\title{
Evaluation of resources for analyzing drug interactions ${ }^{*}+E \mathrm{C}$
}

\author{
Risha I. Patel, PharmD; Robert D. Beckett, PharmD, BCPS
}

See end of article for authors' affiliations.

DOI: http://dx.doi.org/10.3163/1536-5050.104.4.007

\begin{abstract}
Objective: The research sought to evaluate seven drug information resources, specifically designed for analyzing drug interactions for scope, completeness, and ease of use, and determine the consistency of content among the seven resources.

Methods: A cross-sectional study was conducted where 100 drug-drug and drug-dietary supplement interactions were analyzed using 7 drug information resources: Lexicomp Interactions module, Micromedex Drug Interactions, Clinical Pharmacology Drug Interaction Report, Facts \& Comparisons eAnswers, Stockley's Drug Interactions (10th edition), Drug Interactions Analysis and Management (2014), and Drug Interaction Facts (2015). The interaction sample was developed based on published resources and peer input. Two independent reviewers gathered data for each interaction from each of the 7 resources using a common form.
\end{abstract}

Results: Eighty-two drug-drug and 18 drug-dietary supplement interactions were analyzed. Scope scores were higher for Lexicomp Interactions (97.0\%), Clinical Pharmacology Drug Interaction Report (97.0\%), and Micromedex Drug Interactions (93.0\%) compared to all other resources $(p<0.05$ for each comparison). Overall completeness scores were higher for Micromedex Drug Interactions (median 5, interquartile range [IQR] 4 to 5) compared to all other resources $(p<0.01$ for each comparison) and were higher for Lexicomp Interactions (median 4, IQR 4 to 5), Facts \& Comparisons eAnswers (median 4, IQR 4 to 5), and Drug Interaction Facts (4, IQR 4 to 5) compared to all other resources, except Micromedex ( $p<0.05$ for each comparison). Ease of use, in terms of time to locate information and time to gather information, was similar among resources. Consistency score was higher for Micromedex (69.9\%) compared to all other resources ( $p<0.05$ for each comparison).

Conclusions: Clinical Pharmacology Drug Interaction Report, Lexicomp Interactions, and Micromedex Drug Interactions scored highest in scope. Micromedex Drug Interactions and Lexicomp Interactions scored highest in completeness. Consistency scores were overall low, but Micromedex Drug Interactions was the highest.

Keywords: Drug-Drug Interactions, Drug-Dietary Supplement Interactions, Pharmacy

Drug-drug and drug-dietary supplement interactions contribute to the development of adverse drug reactions, which annually cost $\$ 5$ to $\$ 7$ billion in the United States [1]. It is estimated that interactions are responsible for $3 \%$ to $5 \%$ of preventable adverse drug reactions in hospitals and contribute to hospital admissions and emergency

\footnotetext{
* Research was performed with no external funding.

t Preliminary results were presented at the American Society of Health-Systems Pharmacists (ASHP) Midyear Clinical Meeting; New Orleans, LA; December 8, 2015.

EC A supplemental appendix is available with the online version of this journal.
}

room visits [2]. The development of adverse drug reactions due to drug interactions can also prolong hospital stay and worsen patient outcomes both in hospital and outpatient settings. According to World Health Organization estimates, at least $60 \%$ of adverse drug reactions are preventable [1].

The profession of pharmacy has continually evolved to support other health care professionals and provide quality health care for patients, partly by minimizing risk for preventable adverse drug events. In 2014, the Joint Commission of Pharmacy Practitioners (JCPP) published a patient care process model for pharmacists [3]. This model comprises five 
steps to guide provision of patient-centered care: collect, assess, plan, implement, and follow-up. In the assess step, patient information, including information on prescription medications and dietary supplements, is critically analyzed for appropriateness, effectiveness, and safety. A key part of this step is recognizing and appropriately resolving potential drug-drug and drug-dietary supplement interactions. The appropriate action and management of interactions during this step is achieved with the use of drug information databases, many of which are available online for pharmacists' ease of use and accessibility. It is vital for medical libraries, academic libraries, and other institutional and corporate collections supporting all health care professionals to provide drug-drug and drug-dietary supplement resources that facilitate evidence-based practice in this area.

Previous studies have been conducted to evaluate online drug information databases for scope, accuracy, and comprehensiveness of information. In one study, fifteen categories of drug information questions, which included drug-drug interactions, were used to determine the highest ranked database based on scope, completeness, and ease of use [4]. Completeness was based on a three-point scale for drug interactions and varied amongst all databases, with Micromedex having the highest score, followed by Clinical Pharmacology and Lexicomp. The authors of that study concluded that, overall, subscription databases provided more accurate and correct information than free databases, with Clinical Pharmacology, Micromedex, Facts \& Comparisons, and Lexicomp being the best online databases. Further studies have also been conducted to evaluate the use of clinical decision support tools in specific subspecialties. One study evaluated the use of such databases for infectious disease therapy, with $8 \%$ of its evaluation questions pertaining to drug-drug interactions [5]. The databases that had a high score of completeness of drug-drug interactions were DailyMed, Medscape Drug Reference, and Micromedex. Authors of the study concluded that despite the need for improvement, references such as online databases are valuable resources for health care professionals.

Anecdotal observations have identified significant variability among resources, particularly those designed for analyzing drug-drug interactions. Thus, it would be beneficial for health care professionals to be aware of the quality and extent of consistency of resources that are commonly available. Since published studies have not primarily focused on evaluating drug interaction resources, the objectives of this study were to (1) evaluate seven drug information resources specifically designed for analyzing drug interactions for scope, completeness, and ease of use, and (2) determine the consistency of content among the seven resources.

\section{METHODS}

This was a cross-sectional study of seven drug information resources commonly used by pharmacists and other health care professionals in inpatient, outpatient, and community settings for analyzing actual and potential drug-drug and drugdietary supplement interactions. The seven resources were the Lexicomp ${ }^{\circledR}$ Interactions module, Micromedex ${ }^{\circledR}$ Drug Interactions, Clinical Pharmacology Drug Interaction Report, Facts \& Comparisons ${ }^{\circledR}$ eAnswers, Stockley's Drug Interactions (Tenth edition), Drug Interactions Analysis and Management (2014), and Drug Interaction Facts $^{\mathrm{TM}}$ (2015) [6-12]. Electronic resources were selected based on results of an informal survey suggesting that the four electronic resources are most commonly used at health care institutions and community pharmacies. Books were selected to recognize that many pharmacies and medical libraries also retain print resources to supplement electronic resources and that then can be used in the event of an emergency.

Based on previous similar studies that evaluated drug information databases for answering general [4] and infectious diseases [5] drug information questions, it was determined that approximately 100 drug-drug and drug-dietary supplement interactions would provide a sufficient sample size to analyze scope, completeness, ease of use, and consistency among resources. Published review articles focusing on clinically relevant interactions [13, 14], a practicebased research report focusing on minimizing clinical impact of drug interactions [15], and a wellrecognized textbook [16] were used to identify an initial list of potential interactions for analysis to be sure that representative interactions were selected.

To ensure clinically relevant interactions were selected into the sample, the initial list was then sent to three independent expert reviewers: one clinical pharmacy specialist in ambulatory care, one drug information specialist, and one community pharmacist. Reviewers were given two weeks and were asked to spend thirty to sixty minutes reviewing the list, to highlight either frequently 
encountered or clinically relevant interactions, note either rarely encountered or irrelevant interactions, and add any interactions that should be considered for inclusion. The goal was to ultimately develop a sample of eighty to ninety drug-drug and ten to twenty drug-dietary supplement interactions. None of the evaluated resources were consulted to generate the initial list or final sample in order to avoid biasing results.

Once the sample of 100 interactions was developed, 2 independent reviewers gathered data for each of the 100 interactions from each of the 7 resources using a common form. Data collected for each interaction included mechanism (i.e., pharmacokinetic or pharmacodynamic means through which the interaction occurs), severity (i.e., stated degree of seriousness of the interaction), clinical effect (i.e., potential patient outcomes if the interaction were to occur), level of documentation (i.e., level of certainty that the interaction will occur), and course of action (i.e., suggested intervention to minimize risk from the interaction). Information had to be explicitly stated in the resource to be counted, meaning that inferences on the part of reviewers were not allowed. Additionally, the number of minutes taken to locate the interaction and gather the information was measured using a stopwatch and recorded. Formal definitions, as well as training using five sample interactions that were not included the sample, were provided to independent reviewers in order to develop a shared, consistent approach. Any discrepancies were resolved by consensus, with a third investigator available to arbitrate if necessary. There were no discrepancies that could not be resolved by consensus. The primary investigator then entered all data into an Excel spreadsheet for analysis. Data were gathered and entered over a onemonth period in fall 2015.

Five endpoints were developed in order to address the study objectives based on similar previous studies $[4,5]$. First, each resource was scored for scope (i.e., does the resource contain an entry for the interaction?) by calculating a percentage of interactions that had an entry for each resource. Second, each resource was scored for completeness (i.e., does the resource contain unambiguous information addressing the component?) in describing mechanism, severity, clinical effects, level of documentation, and course of action by calculating the percentage of interactions that described each component individually. Additionally, an overall completeness score was calculated by summing the scores of the five components by allotting each component one possible point. For example, if an interaction entry contained unambiguous information describing mechanism, clinical effects, and course of action but did not address severity or level of documentation, it would receive a score of three of five. Mean time to locate and gather the information was determined for each resource to assess ease of use. Finally, to determine level of consistency among resources, the primary investigator reviewed the data gathered by independent reviewers for each interaction and determined whether the information was consistent or partially consistent with findings from the majority of resources. Consistency scores were calculated using the percentage of interactions that were scored as consistent for each resource. If an interaction was not provided by a resource, it was not included when assessing completeness, ease of use, or consistency, as this was assessed in the scope score.

Percentage was used to describe scoring results for scope, completeness, and consistency, except for the overall completeness scores, which were described using median and interquartile range (IQR). Mean and standard error of the mean (SEM) were used to describe results for ease of use. Because the same interactions were assessed using each resource, paired tests were used to conduct inferential statistics. The McNemar test was used to compare scope and consistency scores between resources in a pairwise manner, except for the overall completeness scores, which were compared using the Wilcoxon signed rank test. Ease of use was compared in a pairwise fashion using the paired Student $t$ test. An alpha value of 0.05 was used to determine statistical significance. Inferential statistics were conducted using IBM ${ }^{\circledR}$ SPSS $^{\circledR}$, version 22.

\section{RESULTS}

The initial search for potential interactions to include on the list resulted in 159 drug-drug and 59 drugdietary supplement interactions. Independent expert reviewers did not identify any additional interactions to consider for inclusion. Based on feedback from these reviewers, the list was reduced to a sample of 100 interactions: 82 drug-drug and 18 drug-dietary supplement. The online only appendix lists the final sample of interactions.

Table 1 shows a full description of study results. Scope scores ranged from $67.0 \%$ (Drug Interaction Analysis and Management) to $97.0 \%$ (Lexicomp 


\begin{tabular}{|c|c|c|c|c|c|c|c|}
\hline & LC & MM & $\mathrm{CP}$ & FC & SDI & DIAM & DIF \\
\hline Scope (\%) & 97.0 & 93.0 & 97.0 & 80.0 & 85.0 & 67.0 & 79.0 \\
\hline Mechanism (\%) & 81.4 & 74.2 & 71.1 & 57.5 & 81.2 & 70.1 & 44.3 \\
\hline Severity (\%) & 100.0 & 100.0 & 99.0 & 97.5 & 1.2 & 3.0 & 100.0 \\
\hline Clinical effect (\%) & 64.9 & 89.2 & 64.9 & 68.8 & 80.0 & 70.1 & 77.2 \\
\hline Level of documentation (\%) & 100.0 & 100.0 & 1.0 & 97.5 & 20.0 & 3.0 & 100.0 \\
\hline Course of action (\%) & 82.5 & 93.5 & 69.1 & 97.5 & 91.8 & 86.6 & 98.7 \\
\hline Overall completeness (median [IQR]) & $4(4$ to 5$)$ & $5(4$ to 5$)$ & 3 (2 to 4$)$ & $4(4$ to 5$)$ & 3 (2 to 3 ) & 2 (2 to 3 ) & $4(4$ to 5$)$ \\
\hline Time to locate (mean [SEM], minutes) & $1.0(0.01)$ & $1.0(0.1)$ & $1.0(0.01)$ & $1.0(0.01)$ & $1.1(0.03)$ & $1.0(0.01)$ & $1.1(0.02)$ \\
\hline Time to gather (mean [SEM], minutes) & $3.5(0.2)$ & $3.3(0.2)$ & $2.9(0.2)$ & $2.4(0.1)$ & $3.3(0.2)$ & $3.1(0.2)$ & $2.8(0.2)$ \\
\hline Consistency (\%) & 52.6 & 69.9 & 35.1 & 45.0 & 42.4 & 43.3 & 46.8 \\
\hline \multicolumn{8}{|c|}{$\begin{array}{l}\text { Abbreviations: } \mathrm{CP}=\text { Clinical Pharmacology Drug Interaction Report; DIAM=Drug Interaction Analysis and Management; DIF=Drug Interaction Facts; } \\
\text { FC=Facts \& Comparisons eAnswers; IQR=interquartile range; LC=Lexicomp Interactions; MM=Micromedex Drug Interactions; SDI=Stockley's Drug } \\
\text { Interactions; } \mathrm{SEM}=\text { standard error of the mean. } \\
\text { Legend: Table } 1 \text { describes the scores for each of the principle endpoints in terms of percentage of interactions that achieved the criterion (for scope, } \\
\text { completeness, and consistency) and mean minutes to perform the skill (for ease of use). Definitions of scoring terms can be located in the "Meth- } \\
\text { ods" section. }\end{array}$} \\
\hline
\end{tabular}

Table 1

Study results for scope, completeness, ease of use, and consistency

Interactions and Clinical Pharmacology Drug Interaction Report). Scope scores were higher for Lexicomp Interactions, Clinical Pharmacology Drug Interaction Report, and Micromedex Drug Interactions $(93.0 \%)$ compared to all other resources $(p<0.05$ for each comparison). Scope scores were lower for Drug Interaction Analysis and Management compared to all other resources $(p<0.01$ for each comparison).

Overall completeness scores ranged from a median of 2 (IQR 2 to 3, Drug Interaction Analysis and Management) to 5 (IQR 4 to 5, Micromedex Drug Interactions). Overall completeness scores were higher for Micromedex Drug Interactions compared to all other resources ( $p<0.01$ for each comparison) and were higher for Lexicomp Interactions (median 4, IQR 4 to 5), Facts \& Comparisons eAnswers (median 4, IQR 4 to 5), and Drug Interaction Facts (4, IQR 4 to 5) compared to all other resources $(p<0.05$ for each comparison), except Micromedex. Overall completeness scores were lower for Drug Interaction Analysis and Management compared to all other resources ( $p<0.001$ for each comparison).

Ease of use, in terms of time to locate information and time to gather information, was similar among resources.

Consistency scores ranged from 35.1\% (Clinical Pharmacology Drug Interaction Report) to $69.9 \%$ (Micromedex Drug Interactions). Consistency scores were higher for Micromedex Drug Interactions compared to all other resources $(p<0.05$ for each comparison) and were higher for Lexicomp
Interactions (52.6\%) compared to Clinical Pharmacology Drug Interaction Report $(p=0.021)$. All other results were similar among resources.

\section{DISCUSSION}

This study identified several resources that consistently scored higher in terms of scope, completeness, and consistency. The highest scoring references in terms of scope were Clinical Pharmacology Drug Interaction Report, Lexicomp Interactions, and Micromedex Drug Interactions, although most resources provided information for at least $80 \%$ of the interaction sample. Additionally, Micromedex Drug Interactions and Lexicomp Interactions scored higher in terms of overall completeness and consistency when compared to Clinical Pharmacology Drug Interaction Report, Facts \& Comparisons eAnswers, Stockley's Drug Interactions, Drug Interactions Analysis and Management, and Drug Interaction Facts. Micromedex was also slightly higher for both completeness and consistency compared to Lexicomp Interactions. As expected, consistency among resources was generally low, with no resource achieving a score above $70 \%$. The similar results for ease of use, including both time to locate the interaction information and gather data, suggested that both the electronic and print resources could be efficiently used.

When completeness was broken down into individual components, several niche benefits of individual resources emerged. Although it was not 
the goal of this study to describe individual components of completeness using inferential statistics, results suggested that Lexicomp Interactions (81.4\%) and Stockley's Drug Interactions (81.2\%) might be the resources of choice for locating the mechanism of an interaction, while Micromedex Drug Interactions (89.2\%) would be the more useful resource for identifying potential clinical effects. Level of documentation was best described in Lexicomp Interactions (100.0\%), Micromedex Drug Interactions (100.0\%), Drug Interaction Facts $(100.0 \%)$, and Facts \& Comparisons eAnswers $(97.5 \%)$, while clear recommended courses of action were most commonly provided in Drug Interaction Facts $(98.7 \%)$, Facts \& Comparisons eAnswers (97.5\%), Micromedex Drug Interactions (93.5\%), and Stockley's Drug Interactions (91.8\%). Potential interaction severity was consistently described in all resources except Stockley's Drug Interactions (1.2\%) and Drug Interactions Analysis and Management $(3.0 \%)$.

This study echoed previous published results. Similar to one prior study, it was determined that Micromedex Drug Interactions and Lexicomp Interactions scored highest for completeness [4]. Results also were similar to the study of infectious diseases-related drug information questions, where Micromedex Drug Interactions scored highest; however, that study also found high scores for DailyMed and Medscape Drug Reference, which are free online databases [5]. It was decided not to include free online databases in this study for several reasons. Free online databases, while convenient to use, are generally not designed specifically for identifying interactions and providing appropriate recommendations for management. As these databases provide free access through the Internet, content might not be tailored for the clinical use that health care professionals need.

Academicians and librarians can use the results of this study to help guide collection management decisions for students and faculty in medical, pharmacy, nursing, and other allied health programs. Results can further be used to guide didactic and experiential teaching on the part of clinical and library faculty. In practice, these results suggest several essential resources for health care professionals based on scope, completeness, and consistency scores, but the niche benefits of different resources in terms of completeness help provide evidence-based justification for maintaining a collection that holds a variety of resources that can address different specific information needs. Such variety can help librarians and clinicians provide higher-quality information that will ultimately improve patient care quality. Results can also help guide clinicians toward the ideal resource that will best answer specific interaction-related drug information questions (e.g., what are the potential clinical effects? what is the recommended course of action?) in time-sensitive situations.

Strengths of this study include originality and clinical relevance. Studies evaluating the various available drug information resources have not been done, to the authors' knowledge, specifically in terms of interactions. The peer-input process utilized in determining which interactions to include in this study was beneficial in helping identify relevant interactions for the study, as evidenced by the fact that the highest scoring resources in terms of scope were at $97.0 \%$.

There were several limitations to this study. Individual skills that might have impacted ease of use and completeness scores included varying familiarity and competency with the resources at baseline; however, it should be noted that all individuals involved in the data collection had recently completed the same drug information course. Data collection was also optimized using training sessions, a standardized process, and a standardized tool. Another limitation was that although the definitions of scope, completeness, and ease of use were based on published studies evaluating drug information resources, the precise methods and data collection tool had not previously been validated. Additionally, overall completeness included mechanism, severity, clinical effects, level of documentation, and course of action, equally weighted to build a score out of a maximum of five points. Other clinicians and health care professionals might weigh the five components differently than in this study, based on professional experience and clinical judgment, or include other components altogether. Future studies, such as a study focusing specifically on dietary supplements or evaluating student and pharmacists' ability to assess and evaluate interactions, should be conducted to provide greater insight on the potential impact of drug information resources on patient care.

\section{ACKNOWLEDGMENTS}

The authors thank Iman Aoude, PharmD student, and Rozette Fawzy, PharmD student, for their assistance with data collection. The authors also 
acknowledge Lynn Fletcher, PharmD, BCADM; Joseph K. Jordan, PharmD, BCPS; and Roy LaBarge, PharmD, BCACP, for their expert review of the initial list of interactions.

\section{REFERENCES}

1. Cochrane Z, Hein D, Gregory PJ. Medication misadventures I: adverse drug reactions. In: Malone PM, Kier KL, Stanovich JE, Malone MJ, eds. Drug information: a guide for pharmacists. 5th ed. New York, NY: McGrawHill; 2013.

2. US Food and Drug Administration. Preventable adverse drug reactions: a focus on drug interactions [Internet]. The Administration [cited 29 Jan 2016].

$<$ http://www.fda.gov/Drugs/

DevelopmentApprovalProcess/DevelopmentResources/

DrugInteractionsLabeling/ucm110632.htm > .

3. American College of Clinical Pharmacy. Pharmacists' patient care process [Internet]. The College [cited 29 Jan 2016]. <https://www.accp.com/docs/positions/misc/ JCPP_Pharmacists_Patient_Care_Process.pdf $>$.

4. Clauson KA, Marsh WA, Polen HH, Seamon MJ, Ortiz BI. Clinical decision support tools: analysis of online drug information databases. BMC Med Inform Dec Making 2007 Mar;7(7). DOI: http://dx.doi.org/10.1186/1472-6947-7-7.

5. Polen HH, Zapantis A, Clauson KA, Jebrock J, Paris M. Ability of online drug databases to assist in clinical decision-making with infectious disease therapies. BMC Infect Dis. 2008 Nov;8(153). DOI: http://dx.doi.org/10. 1186/1471-2334-8-153.

6. Wolters Kluwer. Lexicomp ${ }^{\circledR}$ Interactions [Internet]. [cited 8 Feb 2016]. <http://online.lexi.com/lco/action/ interact $>$.

7. Truven Health Analytics. Micromedex ${ }^{\circledR}$ Solutions Drug Interactions [Internet]. [cited 8 Feb 2016]. <http://www. micromedexsolutions.com $>$.

8. Elsevier/Gold Standard. Clinical pharmacology drug interaction report [Internet]. [cited 8 Feb 2016]. <http:// www.clinicalpharmacology-ip.com/Forms/Reports/ intereport.aspx $>$.

9. Wolters Kluwer Health. Facts \& Comparisons ${ }^{\circledR}$ eAnswers [Internet]. [cited 8 Feb 2016]. <http://online. factsandcomparisons.com $>$.
10. Baxter K, Preston CL, eds. Stockley's drug interactions. 10th ed. London, UK: Pharmaceutical Press; 2013.

11. Hansten PD, Horn JR. Drug interactions analysis and management. St. Louis, MO: Wolters Kluwer Health; 2014.

12. Tatro DS, ed. Drug interaction facts 2015. St. Louis, MO: Wolters Kluwer Health; 2014.

13. Bryant L, Fishman T. Clinically important drug-drug interactions and how to manage them. J Prim Health Care. 2009 Jun;1(2):150-1.

14. Haller CA. Clinical approach to adverse events and interactions related to herbal and dietary supplements. Clin Toxicol. 2006 Oct;44(5):605-10.

15. Malone DC, Abarca J, Hansten PD, Grizzle AJ, Armstrong EP, Van Bergen RC, Duncan-Edgar BS, Solomon SL, Lipton RB. Identification of serious drugdrug interactions: results of the partnership to prevent drug-drug interactions. J Am Pharm Assoc. 2004 MarApr;44(2):142-51.

16. McQueen CE, Orr KK. Natural products. In: Krinsky DL, Berardi RR, eds. Handbook of nonprescription drugs: an interactive approach to self-care. 17th ed. Washington, DC: American Pharmacists Association; 2012. p. 9671006.

\section{AUTHORS' AFFILIATIONS}

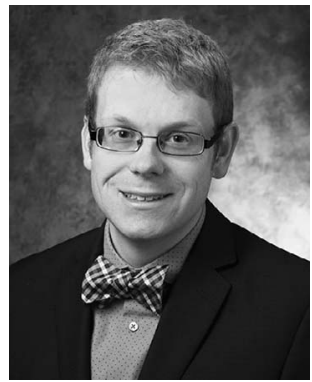

Risha I. Patel, PharmD, ripatel2016@spartans.manchester. edu; PGY1 Community Pharmacy Resident, El Rio Community Health Center, University of Arizona College of Pharmacy, Tucson, AZ; Robert D. Beckett, PharmD, BCPS, rdbeckett@manchester.edu; Director of the Drug Information Center and Assistant Professor of Pharmacy Practice; Manchester University College of Pharmacy, Natural, and Health Sciences, 10627 Diebold Road, Fort Wayne, IN 46845

Received February 2016; accepted May 2016 